\title{
Mild antagonistic effect of Valproic acid in combination with AZD2461 in MCF-7 breast cancer cells
}

\author{
Saman Sargazi ${ }^{1}$, Omid Kooshkaki ${ }^{2}$, Javad Zavar Reza ${ }^{3}$, Ramin Saravani*1,4, Hossein Zarei Jaliani ${ }^{5}$, \\ Shekoufeh Mirinejad ${ }^{1}$, Fatemeh Meshkini ${ }^{6}$
}

Received: 29 Jul 2018

Published: 10 Apr 2019

\begin{abstract}
Background: Breast cancer (BC) is a complex disease, but current treatments are not efficient enough considering increased relapse and decreased survival rate among patients. Poly (ADP-ribose) polymerase inhibitors are recently developed anticancer agents which target cells with defects in homologous recombination (HR) pathway. This study wishes to assess whether the combination of AZD2461 as a newly developed PARP1 inhibitor and valproic acid (VPA), a histone deacetylase inhibitor could effectively reduce the growth of MCF-7 cells with no fundamental DNA repair defect.

Methods: Both trypan blue dye exclusion assay and MTT viability test were used to evaluate cell death. $\gamma$-H2AX levels, as a marker of DNA repair, were measured using in cell ELISA method. The Student's t-test and non-parametric analysis of variance (ANOVA) were applied for our data analyses where $p$-value $<0.05$ was considered statistically significant.

Results: As calculated by CompuSyn software, IC50 values for VPA and AZD2461 were $4.89 \mathrm{mM}$ and $42.83 \mu \mathrm{M}$ respectively following 48 hours treatment. Also, the trypan blue exclusion assay results showed a concentration- and time-dependent decrease when MCF-7 cells were treated with both agents $(\mathrm{p}<0.05)$. Combination analysis showed a mild antagonism $(\mathrm{CI}>1.1)$ while $\gamma$-H2AX levels found not to be significantly increased in MCF-7 cells co-treated with VPA+AZD2461 compared to each agent alone ( $\mathrm{p}=0.29)$.

Conclusion: Our findings revealed that the combination of VPA and AZD2461 could decrease cell viability of MCF-7 cells, but it was not able to significantly increase unrepaired DNA damage sites. The mechanism responsible for drugs combination was not of synergism or addition. Determining the type of involved cell death mechanisms might be followed in further studies.
\end{abstract}

Keywords: Breast cancer, Valproic acid, Combination therapy, AZD2461

Conflicts of Interest: None declared

Funding: Shahid Sadoughi University of Medical Sciences

\section{*This work has been published under CC BY-NC-SA 1.0 license.}

Copyright $₫$ Iran University of Medical Sciences

Cite this article as: Sargazi S, Kooshkaki O, Zavar Reza J, Saravani R, Zarei Jaliani H, Mirinejad Sh, Meshkini F. Mild antagonistic effect of Valproic acid in combination with AZD2461 in MCF-7 breast cancer cells. Med J Islam Repub Iran. 2019 (10 Apr);33:29. https://doi.org/10.47176mjiri.33.29

\section{Introduction}

Breast cancer $(\mathrm{BC})$ is the most frequent female tumor worldwide (1). The range of mortality rates due to this
Corresponding author: Dr Ramin Saravani, saravaniramin@zaums.ac.ir

1. Cellular and Molecular Research Center, Zahedan University of Medical Sciences, Zahedan, Iran

2. Department of Immunology, School of Medicine, Birjand University of Medical Sciences, Birjand, Iran

3. Department of Clinical Biochemistry, School of Medicine, Shahid Sadoughi University of Medical Sciences, Yazd, Iran

4. Department of Clinical Biochemistry, School of Medicine, Zahedan University of Medical Sciences, Zahedan, Iran

5. Protein Engineering Laboratory, Department of Medical Genetics, School of Medicine, Shahid Sadoughi University of Medical Sciences, Yazd, Iran

6. Student Research committee, Shahid Sadoughi University of Medical Sciences, Yazd, Iran $\uparrow$ What is "already known" in this topic:

Basically, combination therapy is regarded as the basis for treatment of multiple diseases, including cancer as promising results were obtained by combining different drugs. Accordingly, (ADP-ribose) polymerase inhibitors were found to act as potent cell-death inducing agents affecting mostly cells with defects in their DNA repair pathways whereas valproic acid is known as a histone-deacetylase inhibitor which proved beneficial in cancer therapy.

\section{$\rightarrow$ What this article adds:}

Showing a mild antagonistic interaction, the combination of VPA and AZD2461 significantly decreased cell viability of MCF-7 cells harboring no mutations in their homologous recombination repair pathway while using this regimen, the unrepaired DNA damage sites was not significantly increased, indicating that AZD2461+VPA could not enhance H2AX phosphorylation. 
type of cancer is approximated up to 6-19 per 100,000 (2). Recent studies have shown that $\mathrm{BC}$ associated mortalities in industrialized countries (i.e., the United States) has been decreased but in developing countries, the disease is still on the rise, especially in Iran. It is also estimated that $\mathrm{BC}$ incidence among Iranian females is almost 10 years earlier than their western counterparts (3). Depending on subtype and stage of $\mathrm{BC}$, there are various strategies to treat it, including Surgery, hormone therapy, radiation therapy, chemotherapy and finally targeted therapy and many patients get more than one type of treatment for the enhancement of their survival (4). There is an increasing number of genes that increase the risk of developing BC when they carry a mutation, such as CTLA4, CYP19A1, FGFR2, ATM, BARD1, BRIP1, CASP8, and TERT(5). Accordingly, one of the crucial reasons for the failure of cancer treatment methods is the presence of one or more of these mutations (6). About $5 \%$ to $10 \%$ of breast cancer patients have a mutation in such important genes (7). For example, BRCA1 (breast cancer responsibility gene 1) and $B R C A 2$ (breast cancer responsibility gene 2 ) are two key genes which their product is involved in repair cell damage response (8) but many tumor cells derived $\mathrm{BC}$ cells do not carry such (i.e., MCF-7 cell line) mutations. Advanced Breast cancers are often attributed to defects in DNA damage response (9), and unrepaired DNA lesions can lead to cancer development and progression (10). Poly (ADP-ribose) polymerases (PARPs) is an enzymatic protein family which is responsible for poly (ADPribose) polymerization and transfer of ADP-ribose to target proteins at the sites of DNA damage (11). Their roles in DNA repair, programmed cell death (PCD), and genomic stability has been well established (12). Contributing to repair of single- or double strand DNA breaks (SSBs and DSBs respectively) is considered to be the most critical functions of this enzyme family consisting of 17 members (13). Recent studies on the field of drug development are focused on using PARP inhibitors (PARPi) in order to perturbate the DNA damage response. Currently, clinical trials suggest that PARP inhibitors may be useful in the treatment of BRCA-related and triple-negative breast cancer (14). Rucaparib (PF-01367338), Niraparib (MK-4827) and Olaparib (AZD-2281) are three classical PARPi which approved by FDA while others are still in early or mid-phases of clinical trials (15). AZD2461 is a novel PARP1, PARP2 and PARP3 inhibitor which initially developed by AstraZeneca but its phase I clinical studies for treating solid malignancies was terminated in 2011 due to efficacy problems (16). As a novel and well tolerated structural analogue for olaparib, AZD2461 displayed high antitumor activity in BC patients (17).

On the other hand, the co-treatment of cancer cells with histone deacetylases (HDACs) inhibitors showed promising advances in the field of cancer therapies (18). Histone deacetylases (which consist of HDAC1 to HDAC11 and sirtuins) are a family of enzymes that remove acetyl groups from histones, allowing them to wrap the DNA tightly (19). HDACs inhibitors (HDACi) selectively inhibit HDACs, lead to reduce chronic inflammation and induction of apoptosis in the intestine and colorectal cancer
(20). Valproic acid (VPA) is a member of HDAC inhibitors that proved efficacious in inhibiting the growth of breast cancer cells alone or in combination with ionizing radiation (IR) in order to sensitize the tumor cells to cell death-inducing agents (21). Recent experiments unraveled the synergism relationship between HDACi and Poly (ADP-ribose) polymerase (PARP) by suppressing cell growth in vitro. For example, triple-negative breast cancers (TNBCs) are conceived to be resistant to PARP inhibitors, but HDAC inhibitors could sensitize TNBC cells to olaparib by down-regulating proteins involved in DNA repair pathways (22). The phosphorylation of histone $\mathrm{H} 2 \mathrm{AX}$ is the first event in response to DNA damage by HDACs and PARPs in cancer cells (23). Hence, the detection of phosphorylated $\mathrm{H} 2 \mathrm{AX}$ can potentially assist the transformation of non-tumor cells to malignant cells. However, the fact that whether the combination of multiple drugs targeting DNA repair pathways could be useful in the treatment of $\mathrm{BC}$ is ill-defined. Previous studies revealed that the combination of PARPi and HDACi induced significantly more DSBs than either agent alone while activating apoptotic cell death mechanisms (24). Although recent studies regarding co-treatment of BRCAdeficient $\mathrm{BC}$ cells with these two inhibitors were shown promising outcomes in the clinic and in vitro, not many $\mathrm{BC}$ cell lines harbor germline mutations in their DNA repair pathways. This indicates the necessity of investigating the possible strategies for combining newly developed specific DNA repair inhibitors and histone deacetylase inhibitors in tumor cells without such genetic backgrounds.

We hypothesized that VPA and AZD2461 together could inhibit the growth of MCF-7 breast cancer cells with no hampered DNA-repair capacity. The current study is conducted to investigate whether the combined use of these inhibitors could improve their antiproliferative and DNA repair efficacy in MCF-7 cell, not carrying such mutations in their profile of DNA repair genes.

\section{Methods}

\section{Chemicals, Cell line and Culture Methods}

MCF-7, human breast (adenocarcinoma) cell line was sourced from Pasteur Institute of Iran and were grown in RPMI 1640 medium obtained from INOCLON (G. Innovative Biotech Co (INOCLON), Iran) supplemented with $10 \%$ FBS (Gibco, Rockville, MD, USA), antibioticantimycotic solution (containing $100 \mathrm{mg} / \mathrm{ml}$ of penicillin, $2.5 \mathrm{mg} / \mathrm{L}$ of amphotericin B, and $100 \mathrm{U} / \mathrm{ml}$ of streptomycin. $0.25 \%$ Trypsin-EDTA solution (G. Innovative Biotech Co (INOCLON), Iran) was used to detach cells from the surface. AZD2461, VPA, Tween 20, Trypan blue and Triton X-100 was procured from Sigma-Aldrich (St. Louis, MO, USA). Cell culture flasks and microtiter plates were supplied by Biofill (Jet Biofill, China). All other chemicals were of certified reagent analytical grade.

\section{MTT Assay}

For evaluation of the viability of MCF-7 cells in response to both drugs alone or combined, MTT assay was performed (25). At first, 6000 cells/well were seeded. Af- 
ter one day, cells were exposed to AZD2461 and VPA at the concentration ranging from $0.5 \mathrm{mM}$ to $16 \mathrm{mM}$ for VPA and $5 \mu \mathrm{M}$ to $160 \mu \mathrm{M}$ for AZD2461. After 24, 48 and 72 hours incubation, $20 \mu \mathrm{L}$ of $5 \mathrm{mg} / \mathrm{ml}$ tetrazolium dye was added to each micro-well and cells were incubated for 3 minutes. Then, the culture was replaced by $200 \mu \mathrm{L}$ of DMSO and kept for 20 minutes in the dark. Using a STAT FAX 2100 microplate reader, the absorbance at $570 \mathrm{~nm}$ wavelength was calculated. The viability percentage was calculated as below mentioned.

Mean OD treatment/Mean OD control $\times 100=\%$

\section{Trypan Blue Dye Exclusion Assay}

The trypan blue dye exclusion assay is used to find the number of viable cells in a cell Suspension (26). Firstly, $50 \mu \mathrm{L}$ of cell suspension was added into a cryo-vial. Then, an equal volume of $0.4 \%$ trypan blue dye was placed to the cell suspension and mixed. Mixture Incubated for at least 3 minutes at room temperature. With the coverslip, one side of a hemacytometer counter filled with the cell suspension and using a conventional light microscope, coloured (death) cells were detected, and therefore the percentage of viable cells was calculated.

\section{H2AX In Cell Elisa Assay}

We used the DuoSet IC ELISA kit (R\&D Systems, Minneapolis, USA) according to the kit manual to measure phosphorylated $\mathrm{H} 2 \mathrm{AX}$ in cell lysates (27). Briefly, the captured antibody was diluted in PBS and immediately coated to a 96 well microplate $(100 \mu \mathrm{L} /$ well $)$ and incubated 24 hours at room temperature. Following 3 times washing, $300 \mu \mathrm{L}$ of block buffer was added and incubated at room temperature for 90 minutes. Then $100 \mu \mathrm{L}$ of sample or standard in IC Diluent added and incubated 2 hours at the same temperature. Then, washing steps were repeated and by placing $100 \mu \mathrm{L}$ of the diluted detection antibody into each well, the plate was again incubated 2 hours at room temperature. Next, $100 \mu \mathrm{L}$ of the diluted Streptavidin-HRP placed into each well before incubating for 20 minutes at the mentioned temperature. In next step after washing, $100 \mu \mathrm{L}$ of substrate solution was added, and after 20 minutes, $50 \mu \mathrm{L}$ of stop solution added to each microwell, and the optical density (OD) of each well was directly measured at $450 \mathrm{~nm}$. The fold changes were calculated by dividing absorbance values (OD) of treated cells by the OD values of adjusted untreated cells in a timedependent manner.

\section{Data Analysis and Statistical methods}

Using SPSS16 software for Windows (release 16, SPSS Inc., Chicago, Illinois), the Student's t-test and nonparametric analysis of variance (ANOVA) were performed for our data analyses when appropriate. In all assays, p-value $<0.05$ was considered significant.

\section{Analysis of Drug Combination}

MCF-7 cells were exposed to both agents (VPA: $0.15 \mathrm{mM}-20 \mathrm{mM}$ and AZD2461: $1.5 \mu \mathrm{M}-200 \mu \mathrm{M})$ with the combined ratio of $1: 115$, each diluted $1: 2$. The drug interactions between constant ratios of both inhibitors were calculated using CompuSyn software (Version 1.0, Combo-Syn In., US) based on Chou-Talalay principles for drugs combination (28) where CI (combination index) values indicate the mechanism of drugs interaction. Briefly, CI $>1.1, \mathrm{CI}=0.9-1.1, \mathrm{CI}<0.9$ represent antagonism, an additive effect, and synergism respectively. The concentration-effect relationships for this combination regimen were plotted using the Median-Effect principles (29).

\section{Results}

Anti-proliferative Effects of VPA, AZD2461 and Their Combination on MCF-7 Cells

As shown in (Figs. 1A and 1B), both agents diminished cell viability of MCF-7 cells in concentration and timedependent manners which was in agreement with the results of trypan blue dye exclusion assay (Figs. 2A and 2B). VPA significantly decreased the number of viable cells following 24 hours $(p=0.005), 48$ hours $(p=0.002)$,
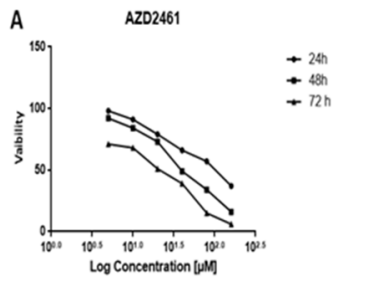

D

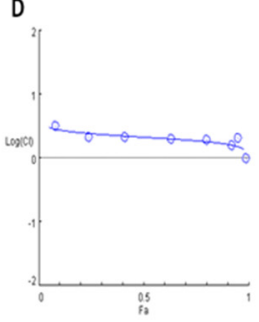

B VPA

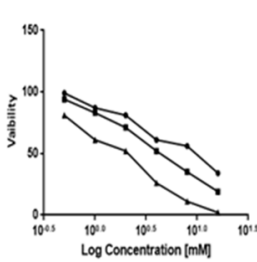

E

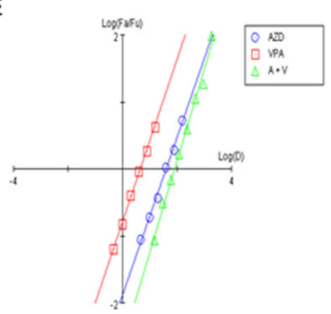

C

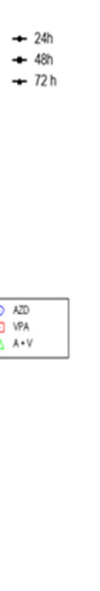

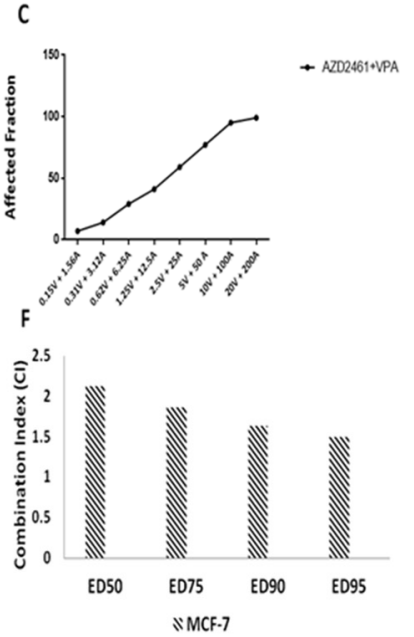

Fig. 1. Concentration-response effects of both inhibitors on MCF-7 cells following 24, 48 and 72 hours of treatment with AZD2461 (A), VPA (B) and their combination (only after $48 \mathrm{~h}$ treatment) (C) depicted by GraphPad prism Software. Combination index plot (D) and median effect-plot (E) for AZD2461+VPA co-treatment are plotted. Various effective doses (EDs) for combining both agents combination demonstrates mild antagonism $(\mathrm{CI}>1.1)(\mathrm{F})$ in MCF-7 cells. 
and 72 hours $(p=0.001)$ of treatment while these $p$-values were $\mathrm{p}=0.008, \mathrm{p}=0.006$, and $\mathrm{p}=0.003$ in cells exposed to AZD2461 after the same periods, respectively.

Analyses of drug combination using combination index and median-effect plots indicate that combination of these two agents would instead exert mild antagonism and not benefits from additive or synergistic interactions (CI>1.1) (Figs. 2D, E). Also, various effective doses (EDs) of VPA and AZD2461 showed CI values higher than 1.1 (Fig. 2F) which indicates the type of drug relationship based on different mechanisms of action. Therefore, the cotreatment of MCF-7 cells with both agents was not sufficiently able to suppress cell proliferation. Table 1 shows the IC50 values calculated by CompuSyn software for both agents alone or in combination.

Effects of Combination with VPA and AZD2461 on $\gamma$ H2AX Levels of MCF-7 Cells

As shown in Figure 3, following $24 \mathrm{~h}$ treatment, the levels of $\gamma-\mathrm{H} 2 \mathrm{AX}$ were significantly increased up to $1.9,2.8$ and 1.7 fold compared to adjusted untreated cells when treating MCF-7 cells with VPA, AZD2461, and their combination, respectively; but co-treatment of two agents did not significantly increase phosphorylated H2AX levels compared to each drug alone $(\mathrm{p}=0.290)$.
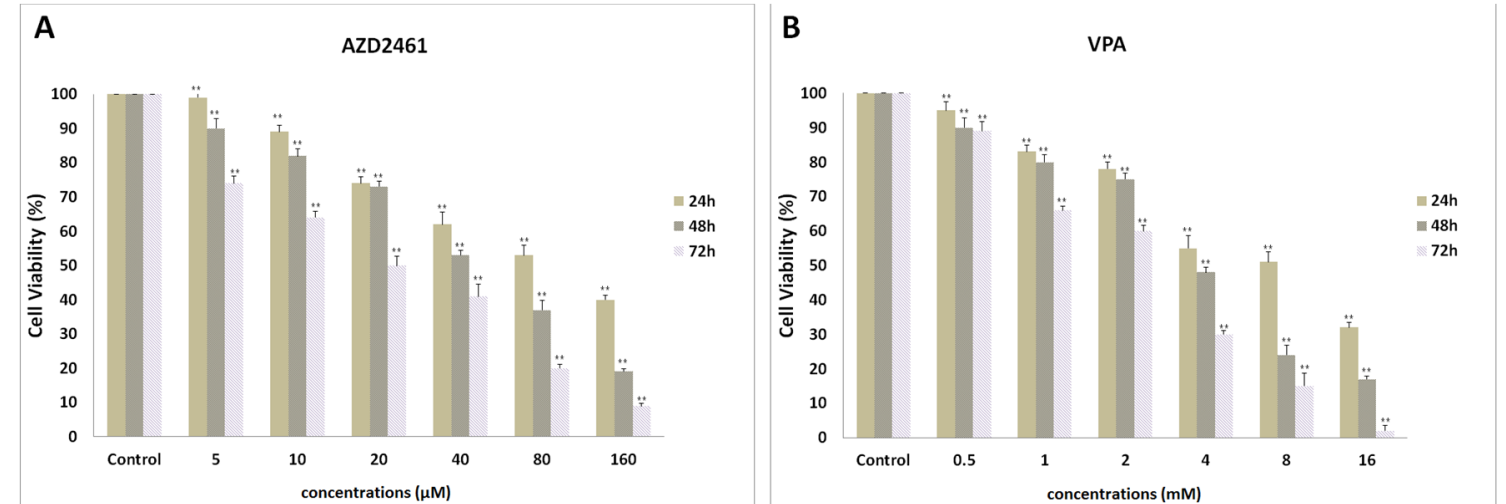

Fig. 2. The effects of AZD2461 (A) and VPA (B) on the viability of MCF-7 cells using trypan blue exclusion assay after 24 to 72 hours treatment $(* * \mathrm{p}<0.05$ compared with control).

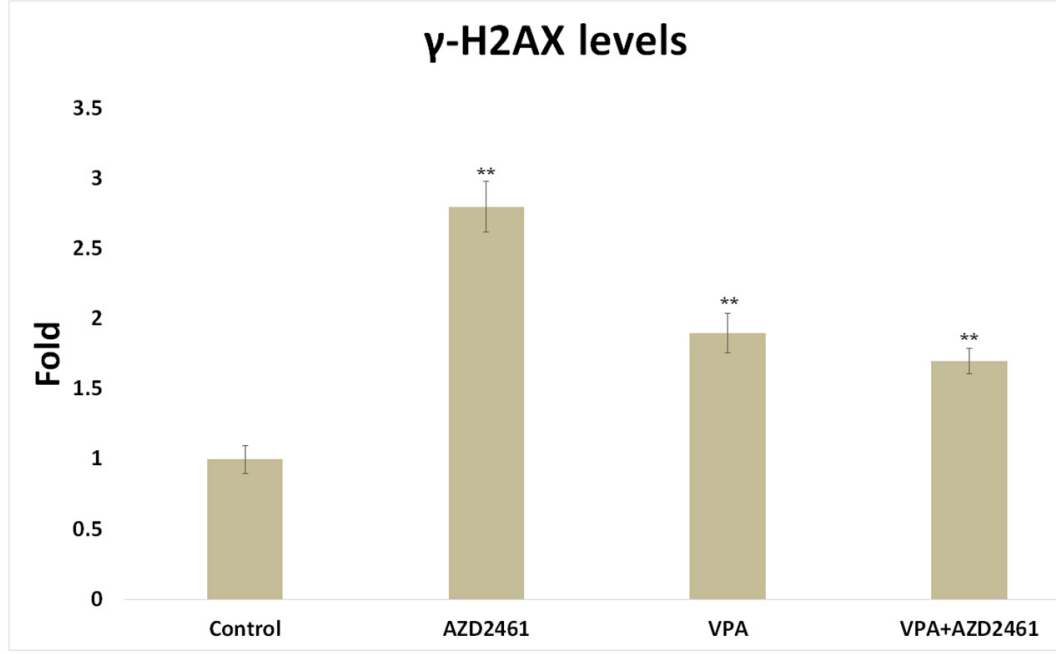

Fig. 3. Analysis of $\gamma$-H2AX following $24 \mathrm{~h}$ treatment with VPA, AZD2461 and their combination. Fold changes were calculated by dividing OD values of treated cells to the OD values of untreated cells. The results indicate that the combination of both agents did not significantly enhance $\mathrm{H} 2 \mathrm{AX}$ phosphorylation of MCF-7 cells $(* * \mathrm{p}<0.05$ compared with adjusted untreated cells). 
therapeutic agent (37). O'Connor and colleagues investigate the efficacy of AZD2461 against olaparib-resistant tumors that overexpress P-glycoprotein, suggesting that use of this specific PARP1-3 inhibitor may have benefits in terms of causing lower drug resistance than olaparib in vitro (38). Konstantinopoulos and colleagues investigated the effects of suberoylanilide hydroxamic acid (SAHA) as an HDACi and olaparib in ovarian cancer cells harboring mutations in BRCA. Based on their findings, SAHA combined with olaparib induced higher apoptosis rates and H2AX loci formation than each other drug alone (39). Phosphorylated H2AX $(\gamma-\mathrm{H} 2 \mathrm{AX})$ is a marker of unrepaired DNA damage that found to be decreased in cells treated with PARPi (40). Also, VPA and PARPi lead to a decrease in mRNA levels of some important DNA repair genes (41).

In the current study, we investigated the combined effects of VPA and AZD2461 in MCF-7 BC cell line besides measuring phosphorylated $\mathrm{H} 2 \mathrm{AX}$ in cell lysates as a marker of DNA damage response perturbation. While many prior studies discovered a synergistic relationship in case of combining other HDACi and inhibitors of PARP family members $(42,43)$, our results did not indicate the probable interaction between VPA and AZD2461. Our data regarding the quantification of phosphorylated $\mathrm{H} 2 \mathrm{AX}$ in MCF-7 cells was also indicative of such mentioned drug interaction. In an experiment conducted by Ha et al. (2014), the synergistic lethal effect of combining ABT888 , a PARPi, in combination with pan-histone deacetylase inhibitor (HDI) and cisplatin was evaluated in human triple negative breast cancer (TNBC) cells (24). The outcomes of this study were in agreement with our findings, suggesting that such co-treatment strategies could prove efficacious in MCF-7 cells irrespective of BRCA1 expression status. This matter is important since a majority of TNBC cells lack germ-line mutations of HR-related factors. To best of our knowledge, the combination of these two drugs in this $\mathrm{BC}$ cell line with this genetic profile did not study before since AZD2461 is known as a recently designed analogue for olaparib. Considering the novelty of combining AZD2461 with other HDACis or classical chemotherapeutic agents, differ ent combinations of strategies may be followed. Our findings support the rationale to use this novel combination against human $\mathrm{BC}$ cells and other malignancies.

\section{Conclusion}

We concluded from our data that although combination regimen of VPA and AZD2461 could decrease cell viability of MCF-7 cells, it was not able to significantly increase unrepaired DNA damage sites and the mechanism responsible for drugs combination was not of synergism or addition. Assessment of other cell death markers, flowcytometric analyses of cell death and evaluating the relative expression of main DNA repair genes and proteins might be followed in further experiments.

\section{Acknowledgements}

This study was financially supported by Shahid
Sadoughi University of Medical Sciences, Yazd, Iran (Grant Number: 5734).

\section{Conflict of Interests}

The authors declare that they have no competing interests.

\section{References}

1. Carla Cedolini, Serena Bertozzi, Ambrogio P. Londero, Sergio Bernardi, Luca Seriau et al. Type of Breast Cancer Diagnosis, Screening, and Survival. Clin Breast Cancer. 2014 Aug;14(4):235-40.

2. Seely JM, Alhassan T. Screening for breast cancer in 2018-what should we be doing today? Curr Oncol. 2018;25(Suppl 1):S115-S124.

3. Bouya S, Koochakzai M, Rafiemanesh H, Balouchi A, Taheri S, Badakhsh $\mathrm{M}$ et al. Health-related quality of life of Iranian breast cancer patients: a meta-analysis and systematic review. Breast Cancer Res Treat. $2018 \mathrm{Jul} ; 170(2): 205-212$.

4. Nayyar A, Gallagher KK, McGuire KP. Definition and Management of Positive Margins for Invasive Breast Cancer. Surg Clin North Am. 2018 Aug;98(4):761-771.

5. Klonowska K, Ratajska M, Czubak K, Kuzniacka A, Brozek I, Koczkowska M, et al. Analysis of large mutations in BARD1 in patients with breast and/or ovarian cancer: the Polish population as an example. Sci Rep. 2015;5:10424.

6. Wu Y, Alvarez M, Slamon DJ, Koeffler P, Vadgama JV. Caspase 8 and maspin are downregulated in breast cancer cells due to $\mathrm{CpG}$ site promoter methylation. BMC Cancer. 2010;10:32.

7. Mehrgou A, Akouchekian M. The importance of BRCA1 and BRCA2 genes mutations in breast cancer development. Med J Islam Repub Iran. 2016;30:369.

8. Ford D, Easton DF, Stratton M, Narod S, Goldgar D, Devilee P, et al. Genetic heterogeneity and penetrance analysis of the BRCA1 and BRCA2 genes in breast cancer families. Am $J$ Hum Genet. 1998;62(3):676-689.

9. Hou L, Chen M, Wang M, Cui X, Gao Y, Xing T, et al. Systematic analyses of key genes and pathways in the development of invasive breast cancer. Gene. 2016 Nov 15;593(1):1-12.

10. Datta A, Brosh RM. New Insights Into DNA Helicases as Druggable Targets for Cancer Therapy. Front Mol Biosci. 2018;5:59.

11. Marijon H, Lee DH, Ding L, Sun H, Gery S, de Gramont A, et al. Co-targeting poly(ADP-ribose) polymerase (PARP) and histone deacetylase (HDAC) in triple-negative breast cancer: Higher synergism in BRCA mutated cells. Biomed Pharmacother. 2018 Mar;99:543-551. doi:10.1016/j.biopha.2018.01.045

12. Comen EA, Robson M. Poly(ADP-Ribose) Polymerase Inhibitors in Triple-Negative Breast Cancer. Cancer J (Sudbury, Mass). 2010;16(1):48-52.

13. Isabelle M, Moreel X, Gagné JP, Rouleau M, Ethier C, Gagné P, et al. "Investigation of PARP-1, PARP-2, and PARG interactomes by affinity-purification mass spectrometry". Proteome Sci. 2010. 8: 22.

14. Lehmann BD, Bauer JA, Chen X, Sanders ME, Chakravarthy AB, Shyr Y, et al. Identification of human triple-negative breast cancer subtypes and preclinical models for selection of targeted therapies. J Clin Invest. 2011;121(7):2750-2767.

15.Zimmer AS, Gillard M, Lipkowitz S, Lee JM. Update on PARP Inhibitors in Breast Cancer. Curr Treat Options Oncol. 2018 Apr $11 ; 19(5): 21$

16. Jaspers JE, Kersbergen A, Boon U, Sol W, van Deemter L, Zander $\mathrm{SA}$, et al. Loss of 53BP1 Causes PARP Inhibitor Resistance in Brca1Mutated Mouse Mammary Tumors. Cancer Discov. 2013 Jan;3(1):6881 .

17. O'Connor OL, Rulten SL, Cranston AN, Odedra R, Brown H, Jaspers JE, et al. The PARP Inhibitor AZD2461 Provides Insights into the Role of PARP3 Inhibition for Both Synthetic Lethality and Tolerability with Chemotherapy in Preclinical Models. Cancer Res. 2016 Oct 15;76(20):6084-6094.

18. Terranova-Barberio M, Thomas S, Ali N, Pawlowska N, Park J, Krings G, et al. HDAC inhibition potentiates immunotherapy in triple negative breast cancer. Oncotarget. 2017;8(69):114156-114172.

19. Li Y, Seto E. HDACs and HDAC Inhibitors in Cancer Development and Therapy. Cold Spring Harb Perspect Med. 2016;6(10):a026831.

20. Bassett SA, Barnett MPG. The Role of Dietary Histone Deacetylases 


(HDACs) Inhibitors in Health and Disease. Nutrients.
2014;6(10):4273-4301.
2014;6(10):4273-4301.

21 Tian Y, Liu G, Wang H, Tian Z, Cai Z, Zhang F, et al. Valproic acid sensitizes breast cancer cells to hydroxyurea through inhibiting RPA2 hyperphosphorylation-mediated DNA repair pathway. DNA Repair. 2017;58:1-12.

22. Papadimitriou M, Mountzios G, Papadimitriou CA. The role of PARP inhibition in triple-negative breast cancer: Unraveling the wide spectrum of synthetic lethality. Cancer Treat Rev. 2018 Jun;67:34-44.

23. Ji J, Zhang Y, Redon CE, Reinhold WC, Chen AP, Fogli LK, et al. Phosphorylated fraction of $\mathrm{H} 2 \mathrm{AX}$ as a measurement for DNA damage in cancer cells and potential applications of a novel assay. PLoS ONE. 2017;12(2): e0171582.

24. Ha K, Fiskus W, Choi DS, Bhaskara S, Cerchietti L, Devaraj SG, et al. Histone deacetylase inhibitor treatment induces 'BRCAness' and synergistic lethality with PARP inhibitor and cisplatin against human triple negative breast cancer cells. Oncotarget. 2014 Jul;5(14):5637.

25. Mosmann T. Rapid colorimetric assay for cellular growth and survival: application to proliferation and cytotoxicity assays. J Immunol Methods. 1983;65(1-2):55-63.

26. Strober W. Trypan blue exclusion test of cell viability. Curr Protoc Immunol. 2001 May; Appendix 3: Appendix 3B.

27. Palla VV, Karaolanis G. Gamma-H2AX: Can it be established as a classical cancer prognostic factor? Tumour Biol. 2017 Mar;39(3).

28. Chou TC. Drug combination studies and their synergy quantification using the Chou-Talalay method. Cancer Res. 2010 Jan 15;70(2):4406.

29. Chou TC. Comparison of dose-effect relationships of carcinogens following low-dose chronic exposure and high-dose single injection: an analysis by the median-effect principle. Carcinogenesis. $1980 \mathrm{Mar}$ 1;1(3):203-13.

30. Madani Tonekaboni SA, Soltan Ghoraie L, Manem VSK, HaibeKains B. Predictive approaches for drug combination discovery in cancer. Brief Bioinform. 2018;19(2):263-276.

31. Yuan Z, Chen S, Sun Q, Wang N, Li D, Miao S, et al. Olaparib hydroxamic acid derivatives as dual PARP and HDAC inhibitors for cancer therapy. Bioorg Med Chem. 2017 Aug 1;25(15):4100-4109.

32. Nolan L, Johnson PW, Ganesan A, Packham G, Crabb SJ. Will histone deacetylase inhibitors require combination with other agents to fulfil their therapeutic potential? Br J Cancer. 2008 Sep;99(5):689.

33. Kuendgen A, Gattermann N. Valproic acid for the treatment of myeloid malignancies. Cancer. 2007 Sep 1;110(5):943-54.

34. Ma X, Wang Y, Gu W, Zhao X. The role and possible molecular mechanism of valproic acid in the growth of MCF-7 breast cancer cells. Croat Med J. 2017;58(5):349-357.

35. Xia Q, Sung J, Chowdhury W, Chen CL, Höti N, Shabbeer S, et al. Chronic Administration of Valproic Acid Inhibits Prostate Cancer Cell Growth In vitro and In vivo. Cancer Res. 2006 Jul 15;66(14):7237-44.

36. Kuendgen A, Schmid M, Schlenk R, Knipp S, Hildebrandt B, Steidl $\mathrm{C}$, et al. The histone deacetylase (HDAC) inhibitor valproic acid as monotherapy or in combination with all-trans retinoic acid in patients with acute myeloid leukemia. Cancer. 2006 Jan 1;106(1):112-9.

37. Rottenberg S, Jaspers JE, Kersbergen A, van der Burg E, Nygren AO, Zander SA, et al. High sensitivity of BRCA1-deficient mammary tumors to the PARP inhibitor AZD2281 alone and in combination with platinum drugs. PNAS. 2008;105(44):17079-17084.

38. O'Connor LO, Rulten SL, Cranston AN, Odedra R, Brown H, Jaspers JE, et al. The PARP Inhibitor AZD2461 Provides Insights into the Role of PARP3 Inhibition for Both Synthetic Lethality and Tolerability with Chemotherapy in Preclinical Models. Cancer Res. 2016 Oct 15;76(20):6084-6094.

39. Konstantinopoulos PA, Wilson AJ, Saskowski J, Wass E, Khabele D. Suberoylanilide Hydroxamic Acid (SAHA) enhances olaparib activity by targeting homologous recombination DNA repair in ovarian cancer. Gynecol Oncol. 2014;133(3):599-606.

40. Ji J, Zhang Y, Redon CE, Reinhold WC, Chen AP, Fogli LK, et al. Phosphorylated fraction of $\mathrm{H} 2 \mathrm{AX}$ as a measurement for DNA damage in cancer cells and potential applications of a novel assay. PLOS ONE. 2017;12(2):e0171582.

41. Ward A, Khanna KK, Wiegmans AP. Targeting homologous recombination, new pre-clinical and clinical therapeutic combinations inhibiting RAD51. Cancer Treat Rev. 2015 Jan 1;41(1):35-45.

42. Chao OS, Goodman OB. Synergistic loss of prostate cancer cell viability by co-inhibition of HDAC and PARP. Mol Cancer Res. 2014
Aug 15:molcanres-0173.

43. Robert C, Nagaria PK, Pawar N, Adewuyi A, Gojo I, Meyers DJ, et al. Histone deacetylase inhibitors decrease NHEJ both by acetylation of repair factors and trapping of PARP1 at DNA double-strand breaks in chromatin. Leuk Res. 2016 Jun 1;45:14-23. 